\title{
In vitro assessment of the cytotoxic effects of novel RGD analogues and conjugates
}

\author{
Anelia Balacheva ${ }^{1}$, Momchil Lambev $^{2}$, Roumyana Detcheva ${ }^{1}$, Thomas Bruckdorfer ${ }^{3}$, Tamara Paypanova ${ }^{4}$ \\ ${ }^{1}$ Roumen Tsanev Institute of Molecular Biology, Bulgarian Academy of Sciences, Bulgaria \\ ${ }^{2}$ Medical University of Varna Prof. Dr. Paraskev Stoyanov, Faculty of Pharmacy, Bulgaria \\ ${ }^{3}$ Iris Biotech GmbH, Germany \\ ${ }^{4}$ Institute of Molecular Biology ”Roumen Tsanev", Bulgarian Academy of Sciences, Bulgaria
}

https://doi.org/10.17952/35EPS.2018.259

\section{Introduction}

The amino acid sequence L-arginyl-glycyl-L-as partic acid (RGD) plays role in interaction of many adhesion proteins by connecting with $\alpha \mathrm{v} \beta 3$ and $\alpha \mathrm{v} \beta 5$ integrin receptors that are overexpressed on various cancer and angiogenic endothelial cells [1]. Synthetic RGD peptides may affect adhesion, and tumour metastasis, or directly induce apoptosis by activating caspases [2].

Delivering peptides with antitumour action directly into cancer cells is one of the most important strategies in recent decades. Drug design based on the RGD structure may provide opportunity for targeted drug delivery and imaging, as well as a new chemotherapeutic treatment for cancer [3].

Herein, we present the synthesis of 9 novel RGD modified peptides and assessment of anti-proliferative activity of some of them.

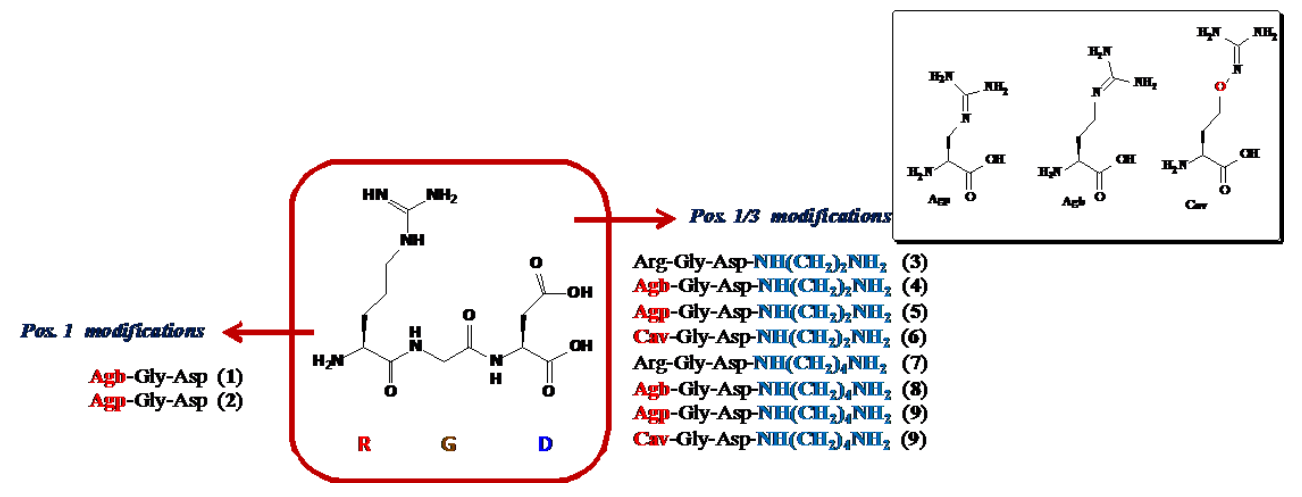

Figure 1: RGD analogues

\section{Results and Discussion}

In order to optimize physicochemical properties (stability, solubility), BBB permeability and cytotoxicity of the parent RGD molecule, the following steps we performed: First we prepared two analogues $(1,2)$ (Fig. 1) containing the shorter homologues of Arg ( $\alpha$-amino-4-guanidino-butyric acid (Agb), and $\alpha$-amino-3guanidino-propionic acid (Agp); Next we synthesized a number of linear RGD analogues modified at 1st position with the arginine mimetics Agb, Agp and Cav, as well as at C-terminus with EDA and DAB (Fig. 1).

All peptides were synthesized using standard solid-phase protocol with the Fmoc/tBu strategy with the help of Activo-P14 synthesizer. For the synthesis of (3-9) commercially available EDA-2-CT Resin and DAB-2-CT Resin was used. The crude peptides were precipitated into cold diethyl ether; the precipitate was dissolved in $10 \% \mathrm{CH}_{3} \mathrm{COOH}$ and desalted by gel filtration on a Sephadex G25. The purity of peptides was analyzed by analytical RP- HPLC and CE. The yields of each product were over 60\%. HPLC and CE analysis revealed a purity of $>95 \%$ for the all peptides.

Next we analyzed the cytotoxic activity of RGD-analogues $(1,2)$ and the parent RGD molecule over two cancer cell lines by colorimetric assay (MTT) in a wide concentration range $(2000-31.5 \mu \mathrm{M})$. The assay was performed on HT-29 and PC-3 cell lines, $72 \mathrm{~h}$ after the treatment. The cells were seeded in a concentration of $3 \times 10^{4}$ cells $/ \mathrm{ml}$. Optical density (OD) was determined at a wavelength of $550 \mathrm{~nm}$ and a reference wavelength of $630 \mathrm{~nm}$ (Fig.2). 

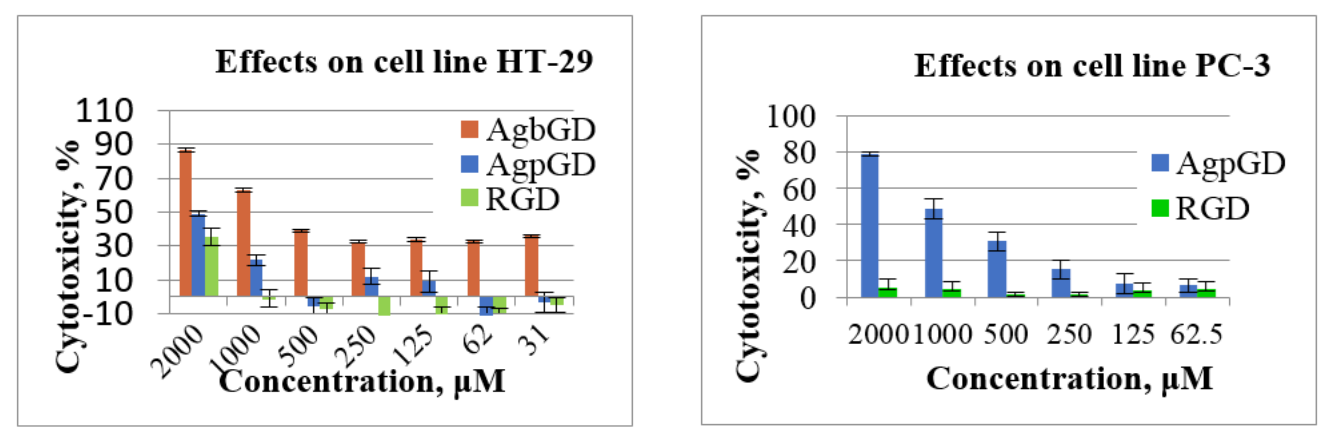

Figure 2: Comparative graph of cytotoxic activity of the tested peptides after $72 \mathrm{~h}$ treatment

We found that the substitution of Arg with its analogues increased the cytotoxic effect of RGD-mimetics in comparison to parent RGD. The highest cytotoxic effect showed AgbGD on HT-29 cells. AgpGD showed significant increase of the cytotoxic effect on PC-3 cells in comparison to parent RGD molecule.

The combination of analgesia and antitumor activity with an absence of toxicity is highly appealing from clinical point of view, and broadens the therapeutic potential and application of RGD mimetics. Therefore, in order to obtain structures of dual-target peptide we synthesized the following endomorhin-2/RGD conjugate (Fig. 3):



Figure 3: Endomorhin-2/RGD conjugate

The cytotoxic activity of the conjugate and the rest of the RGD analogues, as well as their analgesic activities are currently under investigation.

\section{Acknowledgments}

The study was supported by National Science Fund, Ministry of Education and Science, Bulgaria; Project, DN19/17, 20.12.2017.

\section{References}

1. Zamora, A., et al. Chem. Med. Chem. 2018, 10.1002/cmdc.201800282.

2. Danhier, F., et al. Mol. Pharmaceutics 2012, 9, 2961-2973.

3. Lee, M., Chem. Soc. Rev. 2018, 47, 28-52. 